\title{
ASYMMETRIC LOCALLY CONVEX SPACES
}

\section{S. COBZAŞ}

Received 14 February 2005 and in revised form 6 June 2005

The aim of the present paper is to introduce the asymmetric locally convex spaces and to prove some basic properties. Among these I do mention the analogs of the EidelheitTuckey separation theorems, of the Alaoglu-Bourbaki theorem on the weak compactness of the polar of a neighborhood of 0 , and a Krein-Milman-type theorem. These results extend those obtained by García-Raffi et al. (2003) and Cobzaş (2004).

\section{Introduction}

Let $X$ be a real vector space. An asymmetric seminorm on $X$ is a positive sublinear functional $p: X \rightarrow[0 ; \infty)$, that is, $p$ satisfies the conditions

(AN1) $p(x) \geq 0$,

(AN2) $p(t x)=t p(x), t \geq 0$

(AN3) $p(x+y) \leq p(x)+p(y)$

for all $x, y \in X$. The function $\bar{p}: X \rightarrow[0, \infty)$, defined by $\bar{p}(x)=p(-x), x \in X$, is another positive sublinear functional on $X$, called the conjugate of $p$, and

$$
p^{s}(x)=\max \{p(x), p(-x)\}, \quad x \in X,
$$

is a seminorm on $X$. The inequalities

$$
|p(x)-p(y)| \leq p^{s}(x-y), \quad|\bar{p}(x)-\bar{p}(y)| \leq p^{s}(x-y)
$$

hold for all $x, y \in X$. If the seminorm $p^{s}$ is a norm on $X$, then we say that $p$ is an asymmetric norm on $X$. This means that, beside (AN1)-(AN3), it satisfies also the condition

(AN4) $p(x)=0$ and $p(-x)=0$ imply that $x=0$.

The pair $(X, p)$, where $X$ is a linear space and $p$ is an asymmetric seminorm on $X$ is called a space with asymmetric seminorm, respectively, a space with asymmetric norm, if $p$ is an asymmetric norm.

In the last years, the properties of spaces with asymmetric norms were investigated in a series of papers, emphasizing similarities as well as differences with respect to the theory of (symmetric) normed spaces, see $[3,5,6,7,12,13,16,17]$. This study was stimulated 
also by their applications in the complexity of algorithms, see $[11,14,18]$. The aim of the present paper is to develop the basic results in the theory of asymmetric locally convex spaces, a natural extension of asymmetric normed spaces and of (symmetric) locally convex spaces as well.

The function $\rho: X \times X \rightarrow[0 ; \infty)$ defined by $\rho(x, y)=p(y-x), x, y \in X$, is an asymmetric semimetric on $X$. Denote by

$$
B_{p}^{\prime}(x, r)=\left\{x^{\prime} \in X: p\left(x^{\prime}-x\right)<r\right\}, \quad B_{p}(x, r)=\left\{x^{\prime} \in X: p\left(x^{\prime}-x\right) \leq r\right\}
$$

the open, respectively closed, ball in $X$ of center $x$ and radius $r>0$. Denoting by

$$
B_{p}^{\prime}=B_{p}^{\prime}(0,1), \quad B_{p}=B_{p}(0,1)
$$

the corresponding unit balls, then

$$
B_{p}^{\prime}(x, r)=x+r B_{p}^{\prime}, \quad B_{p}(x, r)=x+r B_{p} .
$$

The unit balls $B_{p}^{\prime}$ and $B_{p}$ are convex absorbing subsets of the space $X$ and $p$ agrees with the Minkowski functional associated to any of them.

An asymmetric seminorm $p$ on $X$ generates a topology $\tau_{p}$ on $X$, having as basis of neighborhoods of a point $x \in X$ the family $\left\{B_{p}^{\prime}(x, r): r>0\right\}$ of open $p$-balls. The family $\left\{B_{p}(x, r): r>0\right\}$ of closed $p$-balls is also a neighborhood basis at $x$ for $\tau_{p}$.

The ball $B_{p}^{\prime}(x, r)$ is $\tau_{p}$-open but the ball $B_{p}(x, r)$ need not to be $\tau_{p}$-closed, as can be seen from the following typical example.

Example 1.1. Consider on $\mathbb{R}$ the asymmetric seminorm $u(\alpha)=\max \{\alpha, 0\}, \alpha \in \mathbb{R}$, and denote by $\mathbb{R}_{u}$ the space $\mathbb{R}$ equipped with the topology $\tau_{u}$ generated by $u$. The conjugate seminorm is $\bar{u}(\alpha)=-\min \{\alpha, 0\}$, and $u^{s}(\alpha)=\max \{u(\alpha), \bar{u}(\alpha)\}=|\alpha|$. The topology $\tau_{u}$, called the upper topology of $\mathbb{R}$, is generated by the intervals of the form $(-\infty ; a), a \in \mathbb{R}$, and the family $\{(-\infty ; \alpha+\epsilon): \epsilon>0\}$ is a neighborhood basis of a point $\alpha \in \mathbb{R}$. The set $(-\infty ; 1)=B_{u}^{\prime}(0,1)$ is $\tau_{u}$-open, and the ball $B_{u}(0,1)=(-\infty ; 1]$ is not $\tau_{u}$-closed because $\mathbb{R} \backslash B_{u}(0,1)=(1 ; \infty)$ is not $\tau_{u}$-open.

Remark 1.2. As can be easily seen, the continuity of a mapping $f$ from a topological space $(T, \tau)$ to $\left(\mathbb{R}, \tau_{u}\right)$ is equivalent to its upper semicontinuity as a mapping from $(T, \tau)$ to $(\mathbb{R},|\cdot|)$.

The topology $\tau_{p}$ is translation invariant, that is, the addition $+: X \times X \rightarrow X$ is continuous, but the multiplication by scalars $\cdot: \mathbb{R} \times X \rightarrow X$ need not be continuous, as it is shown by some examples, as, for example, that given in [5]. We will present another one in the context of Example 1.1.

Example 1.3. In the space $(\mathbb{R}, u)$ from Example 1.1 , the interval $(-\infty ; 1 / 2)$ is a $\tau_{u}$ neighborhood of $0=(-1) 0$ but for any $\alpha, \beta>0$, the neighborhood $(-\infty ;-1+\alpha) \times(-\infty$, $\beta)$ of $(-1,0)$ contains the point $(-1,-1)$ and $(-1)(-1)=1 \notin(-\infty ; 1 / 2)$.

The discontinuity of the multiplication by scalars, $(\alpha, x) \mapsto \alpha x$, for $\alpha=-1$ follows also from the fact that the interval $(-\infty ; a)$ is $\tau_{u}$-open but $-(-\infty ; a)=(-a ; \infty)$ is not $\tau_{u}$-open. 
The following proposition will be useful in the study of the continuity of linear mappings between asymmetric locally convex spaces.

Proposition 1.4. If $X$ is a real vector space, $f, g: X \rightarrow \mathbb{R}$ are sublinear functionals, and $\alpha, \beta>0$, then the following conditions are equivalent:

$$
\begin{aligned}
& \forall x \in X, \quad g(x) \leq \beta \Longrightarrow f(x) \leq \alpha, \\
& \forall x \in X, \quad u(f(x)) \leq \frac{\alpha}{\beta} u(g(x)) .
\end{aligned}
$$

If $g(x) \geq 0$ for all $x \in X$, then these two conditions are also equivalent to the following one:

$$
\forall x \in X, \quad f(x) \leq \frac{\alpha}{\beta} g(x) .
$$

Proof. (1.6) $\Rightarrow(1.7)$. Let $x \in X$. If $g(x) \leq 0$, then $g(n x)=n g(x) \leq 0<\alpha, n \in \mathbb{N}$, so that $n f(x)=f(n x) \leq \beta, n \in \mathbb{N}$, implying that $f(x) \leq 0$ and

$$
u(f(x))=0=\frac{\alpha}{\beta} u(g(x)) .
$$

If $g(x)>0$, then $g((\beta / g(x)) x)=\beta$, so that

$$
f\left(\frac{\beta}{g(x)} x\right) \leq \alpha \Longleftrightarrow f(x) \leq \frac{\alpha}{\beta} g(x) \Longleftrightarrow u(f(x)) \leq \frac{\alpha}{\beta} u(g(x)) .
$$

(1.7) $\Rightarrow(1.6)$. Let $x \in X$. If $g(x) \leq 0<\beta$, then $u(g(x))=0$, so that

$$
f(x) \leq u(f(x)) \leq \frac{\alpha}{\beta} u(g(x))=\frac{\alpha}{\beta} g(x) \leq \alpha .
$$

If $g(x)>0$, then by hypothesis,

$$
f(x) \leq u(f(x)) \leq \frac{\alpha}{\beta} u(g(x))=\frac{\alpha}{\beta} g(x) \leq \alpha .
$$

Since $g(x) \geq 0, x \in X$, implies that $u(g(x))=g(x), x \in X$, the equivalence (1.7) $\Leftrightarrow(1.8)$ is obvious.

Let now $P$ be a family of asymmetric seminorms on a real vector space $X$. Denote by $\mathscr{F}(P)$ the family of all nonempty finite subsets of $P$, and for $F \in \mathscr{F}(P), x \in X$, and $r>0$, let

$$
\begin{aligned}
& B_{F}(x, r)=\{y \in X: p(y-x) \leq r, p \in F\}=\bigcap\left\{B_{p}(x, r): p \in F\right\}, \\
& B_{F}^{\prime}(x, r)=\{y \in X: p(y-x)<r, p \in F\}=\bigcap\left\{B_{p}^{\prime}(x, r): p \in F\right\}
\end{aligned}
$$

denote the closed, respectively, open multiball of center $x$ and radius $r$. It is immediate that these multiballs are convex absorbing subsets of $X$. 


\section{Putting}

$$
p_{F}(x)=\max \{p(x): p \in F\}, \quad x \in X,
$$

then $p_{F}$ is an asymmetric seminorm on $X$ and

$$
B_{F}(x, r)=B_{p_{F}}(x, r), \quad B_{F}^{\prime}(x, r)=B_{p_{F}}^{\prime}(x, r) .
$$

The asymmetric locally convex topology associated to the family $P$ of asymmetric seminorms on a real vector space $X$ is the topology $\tau_{P}$ having as basis of neighborhoods of any point $x \in X$ the family $\mathcal{N}^{\prime}(x)=\left\{B_{F}^{\prime}(x, r): r>0, F \in \mathscr{F}(P)\right\}$ of open multiballs. The family $\mathcal{N}(x)=\left\{B_{F}(x, r): r>0, F \in \mathscr{F}(P)\right\}$ of closed multiballs is also a neighborhood basis at $x$ for $\tau_{P}$.

It is easy to check that the family $\mathcal{N}^{\prime}(x)$ fulfills the requirements of a neighborhood basis, that is,

(BN1) $x \in B_{F}^{\prime}(x, r)$,

(BN2) for $B_{F_{1}}^{\prime}\left(x, r_{1}\right)$ and $B_{F_{2}}^{\prime}\left(x, r_{2}\right)$ in $\mathcal{N}^{\prime}(x)$, there exists $B_{F}^{\prime}(x, r) \in \mathcal{N}^{\prime}(x)$ such that $B_{F}^{\prime}(x, r) \subset B_{F_{1}}^{\prime}\left(x, r_{1}\right) \cap B_{F_{2}}^{\prime}\left(x, r_{2}\right)$.

For (BN2), one can take $F=F_{1} \cup F_{2}$ and $r=\min \left\{r_{1}, r_{2}\right\}$.

Obviously, for $P=\{p\}$, we obtain the topology $\tau_{p}$ of an asymmetric seminormed space $(X, p)$ considered above, that is, $\tau_{\{p\}}=\tau_{p}$.

The topology $\tau_{P}$ is derived from a quasiuniformity $\mathcal{W}_{P}$ on $X$ having as vicinities the sets

$$
W_{F}(\epsilon)=\{(x, y) \in X \times X: p(y-x)<\epsilon, p \in F\}
$$

for $F \in \mathscr{F}(P)$ and $\epsilon>0$. Replacing the sign $<$ by $\leq$ in the above definition, the corresponding sets will form a basis for the same quasiuniformity $W_{P}$. A good source for the properties of quasiuniform spaces is the book [10] (see also [4]). Quasiuniform structures related to asymmetric normed spaces were investigated in $[1,2,9]$.

We say that the family $P$ is directed if for any $p_{1}, p_{2} \in P$, there exists $p \in P$ such that $p \geq$ $p_{i}, i=1,2$, where $p \geq q$ stands for the pointwise ordering: $p(x) \geq q(x)$ for all $x \in X$. If the family $P$ is directed, then for any $\tau_{P}$-neighborhood of a point $x \in X$, there exist $p \in P$ and $r>0$ such that $B_{p}^{\prime}(x, r) \subset V$ (resp., $\left.B_{p}(x, r) \subset V\right)$. Indeed, if $B_{F}^{\prime}(x, r) \subset V$, then there exists $p \in P$ such that $p \geq q$ for all $q \in F$ so that $B_{p}^{\prime}(x, r) \subset B_{F}^{\prime}(x, r) \subset V$. Similarly, the vicinities defined by (1.16) with $F=\{p\}, p \in P$, and $\epsilon>0$ form a basis for the quasiuniformity $W_{P}$.

The family

$$
P_{d}=\left\{p_{F}: F \in \mathscr{F}(P)\right\}
$$

where $p_{F}$ is defined by (1.14), is a directed family of asymmetric seminorms generating the same topology as $P$, that is, $\tau_{P_{d}}=\tau_{P}$. Therefore, without restricting the generality, we can always suppose that the family $P$ of asymmetric seminorms is directed. 
Because $B_{F}(x, r)=x+B_{F}(0, r)$ and $B_{F}^{\prime}(x, r)=x+B_{F}^{\prime}(0, r)$, the topology $\tau_{P}$ is translation invariant,

$$
\mathscr{V}(x)=\{x+V: V \in \mathscr{V}(0)\}
$$

where by $\mathscr{V}(x)$ we have denoted the family of all neighborhoods with respect to $\tau_{P}$ of a point $x \in X$.

The addition $+: X \times X \rightarrow X$ is continuous. Indeed, for $x, y \in X$ and the neighborhood $B_{F}^{\prime}(x+y, r)$ of $x+y$, we have $B_{F}^{\prime}(x, r / 2)+B_{F}^{\prime}(y, r / 2) \subset B_{F}^{\prime}(x+y, r)$.

As we have seen in Example 1.3, the multiplication by scalars need not be continuous, even in asymmetric seminormed spaces.

The topology $\tau_{p}$ generated by an asymmetric norm is not always Hausdorff. A necessary and sufficient condition in order that $\tau_{p}$ be Hausdorff is given in the following proposition.

Proposition 1.5 (see [13]). For an asymmetric seminorm $p$ on a real vector space $X$, put

$$
\tilde{p}(x)=\inf \left\{p\left(x^{\prime}\right)+p\left(x^{\prime}-x\right): x^{\prime} \in X\right\}, \quad x \in X .
$$

(1) The functional $\tilde{p}$ is a (symmetric) seminorm on $X, \tilde{p} \leq p$, and $\tilde{p}$ is the greatest of the seminorms on $X$ majorized by $p$.

(2) The topology $\tau_{p}$ generated by $p$ is Hausdorff if and only if $\tilde{p}(x)>0$ for every $x \neq 0$.

Proof. We will give a proof of the first assertion, different from that given in [13]. The second assertion will be proved in the more general context of asymmetric locally convex spaces.

First, observe that replacing $x^{\prime}$ by $x^{\prime}-x$ in (1.19), we get

$$
\begin{aligned}
\tilde{p}(-x) & =\inf \left\{p\left(x^{\prime}\right)+p\left(x^{\prime}+x\right): x^{\prime} \in X\right\} \\
& =\inf \left\{p\left(x^{\prime}-x\right)+p\left(\left(x^{\prime}-x\right)+x\right): x^{\prime} \in X\right\}=\tilde{p}(x),
\end{aligned}
$$

so that $\tilde{p}$ is symmetric. The positive homogeneity of $\tilde{p}, \tilde{p}(\alpha x)=\alpha \tilde{p}(x), x \in X, \alpha \geq 0$, is obvious. For $x, y \in X$ and arbitrary $x^{\prime}, y^{\prime} \in X$, we have

$$
\tilde{p}(x+y) \leq p\left(x^{\prime}+y^{\prime}\right)+p\left(x^{\prime}+y^{\prime}-x-y\right) \leq p\left(x^{\prime}\right)+p\left(x^{\prime}-x\right)+p\left(y^{\prime}\right)+p\left(y^{\prime}-y\right),
$$

so that passing to infimum with respect to $x^{\prime}, y^{\prime} \in X$, we obtain the subadditivity of $\tilde{p}$,

$$
\tilde{p}(x+y) \leq \tilde{p}(x)+\tilde{p}(y) .
$$

Suppose now that there exists a seminorm $q$ on $X$ such that $q \leq p$, that is, for all $z \in X$, $q(z) \leq p(z)$, and $\tilde{p}(x)<q(x) \leq p(x)$, for some $x \in X$. Then, by the definition of $\tilde{p}$, there exists $x^{\prime} \in X$ such that $\tilde{p}(x)<p\left(x^{\prime}\right)+p\left(x^{\prime}-x\right)<q(x)$, leading to the contradiction

$$
q(x) \leq q\left(x^{\prime}\right)+q\left(x-x^{\prime}\right)=q\left(x^{\prime}\right)+q\left(x^{\prime}-x\right) \leq p\left(x^{\prime}\right)+p\left(x^{\prime}-x\right)<q(x) .
$$


The following characterization of the Hausdorff separation property for locally convex spaces is well known, see, for example, [19, Lemma VIII.1.4].

Proposition 1.6. Let $(X, Q)$ be a locally convex space, where $Q$ is a family of seminorms generating the topology $\tau_{Q}$ of $X$. The topology $\tau_{Q}$ is Hausdorff separated if and only if for every $x \in X, x \neq 0$, there exists $q \in Q$ such that $q(x)>0$.

In the case of asymmetric locally convex spaces, we have the following characterization.

Proposition 1.7. Let $P$ be a family of asymmetric seminorms on a real vector space $X$. The asymmetric locally convex topology $\tau_{P}$ is Hausdorff separated if and only if for every $x \in X, x \neq 0$, there exists $p \in P$ such that $\tilde{p}(x)>0$, where $\tilde{p}$ is the seminorm associated to the asymmetric seminorm $p$ through the formula (1.19).

Proof. Suppose that $P$ is directed and let

$$
\tilde{P}=\{\tilde{p}: p \in P\} .
$$

Denote by $\tau_{\tilde{P}}$ the locally convex topology on $X$ generated by the family $\tilde{P}$ of seminorms. The topology $\tau_{P}$ is finer than $\tau_{\tilde{P}}$. Indeed, $\tilde{G} \in \tau_{\tilde{P}}$ is equivalent to the fact that for every $x \in \tilde{G}$, there exist $p \in P$ and $r>0$ such that $B_{\tilde{p}}^{\prime}(x, r) \subset \tilde{G}$. Because $p(y-x)<r$ implies that $\tilde{p}(y-x) \leq p(y-x)<r$, we have $B_{p}^{\prime}(x, r) \subset B_{\tilde{p}}^{\prime}(x, r) \subset \tilde{G}$, so that $\tilde{G} \in \tau_{P}$. If for every $x \in X, x \neq 0$, there exists $p \in P$ such that $\tilde{p}(x)>0$, then the locally convex topology $\tau_{\tilde{P}}$ is separated Hausdorff, and so will be the finer topology $\tau_{P}$.

Conversely, suppose that the topology $\tau_{P}$ is Hausdorff and show that $\tilde{p}(x)=0$ for all $p \in P$ implies that $x=0$.

Let $x \in P$ be such that $\tilde{p}(x)=0$ for all $p \in P$. By the definition (1.19) of the seminorm $\tilde{p}$, for every $p \in P$ and $n \in \mathbb{N}$, there exists an element $x_{(p, n)} \in X$ such that

$$
p\left(x_{(p, n)}\right)+p\left(x_{(p, n)}-x\right)<\frac{1}{n} .
$$

Define the order on $P \times \mathbb{N}$ by $(p, n) \leq(q, m)$ if and only if $p \leq q$ and $n \leq m$. Since the family $P$ of asymmetric seminorms is directed, the set $P \times \mathbb{N}$ is also directed with respect to the order we just defined. Therefore, $\left\{x_{(p, n)}:(p, n) \in P \times \mathbb{N}\right\}$ is a net in $X$ and by (1.25), we have

$$
p\left(x_{(p, n)}\right)<\frac{1}{n}, \quad p\left(x_{(p, n)}-x\right)<\frac{1}{n},
$$

for all $(p, n) \in P \times \mathbb{N}$.

We will prove that the net $\left\{x_{(p, n)}\right\}$ converges to both 0 and $x$. Since the topology $\tau_{P}$ is Hausdorff, this will imply that $x=0$.

To prove that the net $\left\{x_{(p, n)}\right\}$ converges to 0 , we have to show that for every $p \in P$, the net $\left\{p\left(x_{(p, n)}\right)\right\}$ tends to 0 , that is,

$$
\begin{gathered}
\forall p \in P, \forall \epsilon>0, \exists\left(p_{0}, n_{0}\right) \in P \times \mathbb{N}, \forall(q, n) \in P \times \mathbb{N}, \text { such that } \\
(q, n) \geq\left(p_{0}, n_{0}\right) \Longrightarrow p\left(x_{(q, n)}\right)<\epsilon .
\end{gathered}
$$


Let $p \in P$ and $\epsilon>0$. Put $p_{0}=p$ and let $n_{0} \in \mathbb{N}$ be such that $1 / n_{0}<\epsilon$. Then for every $(q, n) \in P \times \mathbb{N}$ such that $q \geq p$ and $n \geq n_{0}$, we have

$$
p\left(x_{(q, n)}\right) \leq q\left(x_{(q, n)}\right)<\frac{1}{n} \leq \frac{1}{n_{0}}<\epsilon .
$$

The convergence of $\left\{p\left(x_{(p, n)}-x\right)\right\}$ to 0 , which is equivalent to the $\tau_{P}$-convergence of $\left\{x_{(p, n)}\right\}$ to $x$, can be proved similarly, using the second inequality in (1.26).

Corollary 1.8. Let $(X, P)$ be an asymmetric locally convex space. If the topology $\tau_{P}$ is Hausdorff, then for every $x \in X, x \neq 0$, there exists $p \in P$ such that $p(x)>0$.

Proof. If the topology $\tau_{P}$ is Hausdorff, then for every $x \in X, x \neq 0$, there exists $p \in P$ such that $\tilde{p}(x)>0$. Replacing $x$ by $-x$ and taking $x^{\prime}=0$ in the definition (1.19) of the seminorm $\tilde{p}$, we get

$$
p(x)=p(0)+p(0+x) \geq \tilde{p}(x)>0 .
$$

As in the symmetric case, asymmetric locally convex topologies can be defined through some basic families of convex absorbing sets.

A nonempty family $\mathscr{C}$ of subsets of a real vector space $X$ is called an asymmetric locally convex basis provided that

(BC1) each $C \in \mathscr{C}$ is convex and absorbing;

(BC2) for every $C_{1}, C_{2} \in \mathscr{C}$, there exists $C \in \mathscr{C}$ such that $C \subset C_{1} \cap C_{2}$;

(BC3) for every $C \in \mathscr{C}$ and $\alpha>0$, there exists $D \in \mathscr{C}$ such that $D \subset \alpha C$.

Define a mapping $\mathcal{U}: X \rightarrow 2^{X}$ by

$$
u(x)=\{U \subset X: \exists C \in \mathscr{C} \text { such that } x+C \subset U\} .
$$

Recall that for an absorbing subset $C$ of $X$, the Minkowski functional $p_{C}: X \rightarrow[0 ; \infty)$ is defined by

$$
p_{C}(x)=\inf \{t>0: x \in t C\}
$$

If $C$ is absorbing and convex, then $p_{C}$ is a positive sublinear functional, and

$$
\left\{x \in X: p_{C}(x)<1\right\} \subset C \subset\left\{x \in X: p_{C}(x) \leq 1\right\} .
$$

Conversely, if $p$ is a positive sublinear functional on $X$, then $C^{\prime}=\{x \in X: p(x)<1\}$ and $C=\{x \in X: p(x) \leq 1\}$ are convex absorbing subsets of $X$, and $p_{C^{\prime}}=p_{C}=p$.

Denoting by

$$
P=\left\{p_{C}: C \in \mathscr{C}\right\}
$$

the family of all Minkowski functionals associated to the sets in $\mathscr{C}$, then $P$ is a family of asymmetric seminorms on $X$. By $(\mathrm{BC} 1)$ and the fact that $p_{C} \leq p_{D}$ if $D \subset C$, it follows that the family $P$ is directed. 
Proposition 1.9. The family $\mathcal{U}(x)$ of subsets of $X$ given by (1.30) satisfies the axioms of a neighborhood system, so that it defines a topology $\tau_{6}$ on $X$. This topology agrees with the asymmetric locally convex topology generated by the family (1.33) of asymmetric seminorms.

Proof. One can easily check that the family $U$ of subsets of $X$ satisfies the axioms of a neighborhood system.

Since both of the topologies $\tau_{\bullet}$ and $\tau_{P}$ are translation invariant, in order to prove their coincidence, it suffices to show that they have the same 0 -neighborhoods. Denote by $\mathscr{V}$ the neighborhood mapping associated to $\tau_{P}$. If $U \in \mathcal{U}(0)$, then there exists $C \in \mathscr{C}$ such that $C \subset U$. The inclusions

$$
\left\{x \in X: p_{C}(x)<1\right\} \subset C \subset U
$$

show that $U \in \mathscr{V}(0)$.

Conversely, if $V \in \mathscr{V}(0)$, then there exist $C \in \mathscr{C}$ and $r>0$ such that $\left\{x \in X: p_{C}(x) \leq\right.$ $r\} \subset V$. By (BC3), there exists $D \in \mathscr{C}$ such that $D \subset r C$. But then

$$
D \subset r C \subset\left\{x \in X: p_{C}(x) \leq r\right\} \subset V
$$

so that $V \in \mathcal{U}(0)$.

\section{Bounded linear mappings between asymmetric locally convex spaces and the dual space}

Let $(X, P),(Y, Q)$ be two asymmetric locally convex spaces with the topologies $\tau_{P}$ and $\tau_{Q}$ generated by the families $P$ and $Q$ of asymmetric seminorms on $X$ and $Y$, respectively. In the following, when we say that $(X, P)$ is an asymmetric locally convex space, we understand that $X$ is a real vector space, $P$ is a family of asymmetric seminorms on $X$, and $\tau_{P}$ is the asymmetric locally convex topology associated to $P$.

A linear mapping $A: X \rightarrow Y$ is called $(P, Q)$-bounded if for every $q \in Q$, there exist $F \in \mathscr{F}(P)$ and $L \geq 0$ such that

$$
\forall x \in X, \quad q(A x) \leq L \max \{p(x): p \in F\}
$$

If the family $P$ is directed, then the $(P, Q)$-boundedness of $A$ is equivalent to the condition that for every $q \in Q$, there exist $p \in P$ and $L \geq 0$ such that

$$
\forall x \in X, \quad q(A x) \leq L p(x)
$$

The continuity of the mapping $A$ from $\left(X, \tau_{P}\right)$ to $\left(Y, \tau_{Q}\right)$ is called $\left(\tau_{P}, \tau_{Q}\right)$-continuity. We will use also the term $(P, Q)$-continuity for this property, and $(P, u)$-continuity in the case of $\left(\tau_{P}, \tau_{u}\right)$-continuous linear functionals.

Because both of the topologies $\tau_{P}$ and $\tau_{Q}$ are translation invariant, we have the following result. Recall that a mapping $F$ between two quasiuniform spaces $(X, \mathcal{U})$ and $(Y, \mathcal{W})$ is called quasiuniformly continuous if for every $W \in \mathcal{W}$, there exists $U \in \mathcal{U}$ such that $(F(x), F(y)) \in W$ for every $(x, y) \in U$. 
Proposition 2.1. Let $(X, P)$ and $(Y, Q)$ be asymmetric locally convex spaces and $A: X \rightarrow Y$ a linear mapping. The following conditions are equivalent.

(1) The mapping $A$ is $(P, Q)$-continuous on $X$.

(2) The mapping $A$ is continuous at $0 \in X$.

(3) The mapping $A$ is continuous at some point $x_{0} \in X$.

The following proposition emphasizes the equivalence of continuity and boundedness for linear mappings.

Proposition 2.2. Let $(X, P)$ and $(Y, Q)$ be two asymmetric locally convex spaces and $A$ : $X \rightarrow Y$ a linear mapping. The following assertions are equivalent.

(1) The mapping $A$ is $(P, Q)$-continuous on $X$.

(2) The mapping $A$ is continuous at $0 \in X$.

(3) The mapping $A$ is $(P, Q)$-bounded.

(4) The mapping $A$ is quasiuniformly continuous with respect to the quasiuniformities $W_{P}$ and $W_{Q}$.

Proof. The equivalence (1) $\Leftrightarrow$ (2) follows from the preceding proposition.

Suppose that the families $P$ and $Q$ are directed.

$(2) \Leftrightarrow(3)$. For $q \in Q$, consider the $\tau_{Q}$-neighborhood $V=B_{q}(0,1)$ of $A 0=0 \in Y$, and let $U$ be a neighborhood of $0 \in X$ such that $A(U) \subset V$. If $p \in P$ and $r>0$ are such that $B_{p}(0, r) \subset U$, then

$$
\forall x \in X, \quad p(x) \leq r \Longrightarrow q(A x) \leq 1
$$

By Proposition 1.4 applied to $f(x)=q(A x)$ and $g(x)=p(x)$, this relation implies that

$$
\forall x \in X, \quad q(A x) \leq \frac{1}{r} p(x)
$$

Conversely, if $A$ is $(P, Q)$-bounded, then for every $\tau_{Q}$-neighborhood $V$ of $0 \in Y$, there exist $q \in Q$ and $R>0$ such that $B_{q}(0, R) \subset V$. Let $p \in P$ and $L \geq 0$ be such that the condition (2.2) is fulfilled. Taking $r:=R /(L+1)$, we have

$$
\forall x \in B_{p}(0, r), \quad q(A x) \leq L p(x) \leq \frac{L}{L+1} R \leq R,
$$

which shows that $A\left(B_{p}(0, r)\right) \subset B_{q}(0, R) \subset V$, that is, $A$ is continuous at $0 \in X$.

The implication $(3) \Rightarrow(4)$ follows from the $(P, Q)$-boundedness of the mapping $A$ and the definition (1.16) of the vicinities.

To prove (4) $\Rightarrow(3)$, suppose that $A$ is $\left(\mathscr{W}_{P}, \mathbb{W}_{Q}\right)$-quasiuniformly continuous. For $q \in Q$, let $W=\left\{\left(y^{\prime}, y\right) \in Y \times Y: q\left(y-y^{\prime}\right) \leq 1\right\} \in \mathcal{W}_{Q}$, and let $U=\left\{\left(x^{\prime}, x\right) \in X \times X: p(x-\right.$ $\left.\left.x^{\prime}\right) \leq r\right\} \in \mathscr{W}_{P}$ be such that $\left(x^{\prime}, x\right) \in U$ implies that $\left(A x^{\prime}, A x\right) \in W$. Taking $x^{\prime}=0$, it follows that

$$
\forall x \in X, \quad p(x) \leq r \Rightarrow q(A x) \leq 1,
$$


so that, by Proposition 1.4,

$$
\forall x \in X, \quad q(A x) \leq \frac{1}{r} p(x)
$$

In the case of linear functionals on an asymmetric locally convex space, we have the following characterization of continuity, where $u$ is as in Example 1.1.

Proposition 2.3. Let $(X, P)$ be an asymmetric locally convex space and $\varphi: X \rightarrow \mathbb{R}$ a linear functional. The following assertions are equivalent.

(1) $\varphi$ is $(P, u)$-continuous at $0 \in X$.

(2) $\varphi$ is $(P, u)$-continuous on $X$.

(3) There exist $p \in P$ and $L \geq 0$ such that

$$
\forall x \in X, \quad \varphi(x) \leq L p(x)
$$

(4) $\varphi$ is upper semicontinuous from $\left(X, \tau_{P}\right)$ to $(\mathbb{R},|\cdot|)$.

Remark 2.4. If the family $P$ is not directed, then the $(P, u)$-continuity of the functional $\varphi$ is equivalent to the condition that there exist $F \in \mathscr{F}(P)$ and $L \geq 0$ such that

$$
\forall x \in X, \quad \varphi(x) \leq L \max \{p(x): p \in F\}=L p_{F}(x) .
$$

The dual of an asymmetric locally convex space. For an asymmetric locally convex space $(X, P)$, denote by $X^{b}=X_{P}^{b}$ the set of all linear $(P, u)$-continuous functionals. If $P=\{p\}$, then we obtain the dual space $X_{p}^{b}$ of an asymmetric normed space $(X, p)$ considered in [13].

Let $X^{\#}$ be the algebraic dual space to $X$, that is, the space of all linear functionals on $X$. In contrast to the symmetric case, $X^{b}=X_{P}^{b}$ is not a subspace of $X^{\#}$, but merely a convex cone, that is,

(i) $\varphi, \psi \in X^{b} \Rightarrow \varphi+\psi \in X^{b}$,

(ii) $\varphi \in X^{b}$ and $\alpha \geq 0 \Rightarrow \alpha \varphi \in X^{b}$.

There are examples in the case $P=\{p\}$ of $p$-bounded linear functionals $\varphi$ on a space with asymmetric norm $(X, p)$ such that $-\varphi$ is not $p$-bounded, see [5]. A simpler example can be exhibited in the space $(\mathbb{R}, u)$ from Example 1.1 .

Example 2.5. The identity mapping $\varphi(t)=t, t \in \mathbb{R}$, is $\left(\tau_{u}, \tau_{u}\right)$-continuous because

$$
\forall t \in \mathbb{R}, \quad \varphi(t)=t \leq \max \{t, 0\}=u(t),
$$

but $-\varphi$ is not $\left(\tau_{u}, \tau_{u}\right)$-continuous, because it is impossible to find $L \geq 0$ such that $(-\varphi)(t) \leq L u(t)$ for all $t \in \mathbb{R}$. Indeed, taking $t=-1$, we obtain the contradiction

$$
1=(-\varphi)(-1) \leq L \cdot u(-1)=0 \text {. }
$$

Remark 2.6. It is easy to check that a linear functional $\varphi(t)=a t, t \in \mathbb{R}$, is $\left(\tau_{u}, \tau_{u}\right)$ continuous if and only if $a \geq 0$. Indeed if $a \geq 0$, then $\varphi(t)=a t \leq u(a t)=a u(t), t \in \mathbb{R}$. If $a<0$, then, reasoning as above, one concludes that $\varphi$ fails to be continuous. 
Suppose that the family $P$ of asymmetric seminorms is directed, and for $p \in P$, let $p^{s}(x)=\max \{p(x), p(-x)\}$ be the symmetric seminorm attached to $p$, and let

$$
P^{s}=\left\{p^{s}: p \in P\right\}
$$

Denote by $X^{*}=\left(X, P^{s}\right)^{*}$ the dual space of the locally convex space $\left(X, P^{s}\right)$. Since for a seminorm $q$ and a linear functional $\varphi$ we have

$$
\forall x \in X, \quad \varphi(x) \leq L q(x) \Longleftrightarrow \forall x \in X, \quad|\varphi(x)| \leq L q(x),
$$

we have $X^{b}=X_{P}^{b} \subset X^{*}=\left(X, P^{s}\right)^{*}$. Indeed, if $\varphi \in X^{b}, p \in P$, and $L \geq 0$ are such that for all $x \in X, \varphi(x) \leq L p(x)$, then, the inequality $p \leq p^{s}$ and the above equivalence imply that $|\varphi(x)| \leq L p^{s}(x), x \in X$, showing that $\varphi \in X^{*}$.

Let $p$ be an asymmetric seminorm on a real vector space $X$ and let $\varphi: X \rightarrow \mathbb{R}$ be a linear functional. Put

$$
\|\left.\varphi\right|_{p}=\sup \varphi\left(B_{p}\right)
$$

We say that the functional $\varphi$ is $p$-bounded if there exists $L \geq 0$ such that

$$
\forall x \in X, \quad \varphi(x) \leq L p(x) .
$$

A number $L \geq 0$ satisfying (2.15) is called a $p$-Lipschitz constant for $\varphi$. The functional $\varphi$ is $p$-bounded if and only if $\|\left.\varphi\right|_{p}<\infty$ and $\|\left.\varphi\right|_{p}$ is the smallest $p$-Lipschitz constant for $\varphi$. The $p$-boundedness of $\varphi$ is also equivalent to its $\left(\tau_{p}, \tau_{u}\right)$-continuity. The functional $\|\left.\cdot\right|_{p}$ defined by (2.14) is an asymmetric norm on the asymmetric dual $X_{p}^{b}$ of $(X, p)$, that is, $\left.\left\|\varphi+\left.\psi\right|_{p} \leq\right\| \varphi\right|_{p}+\left.\left\|\left.\psi\right|_{p},\right\| \alpha \varphi\right|_{p}=\alpha \|\left.\varphi\right|_{p}$, for all $\varphi, \psi \in X_{p}^{b}$ and $\alpha \geq 0$. Also, $\|\left.\varphi\right|_{p}>0$ for $\varphi \in X_{p}^{b} \backslash\{0\}$.

Similar considerations can be done with respect to the conjugate asymmetric seminorm $\bar{p}(x)=p(-x)$ of $p$ and

$$
\|\left.\varphi\right|_{\bar{p}}=\sup \varphi\left(B_{\bar{p}}\right)
$$

Some properties of the norm $\|\left.\cdot\right|_{p}$ are collected in the following proposition.

PRoposition 2.7. Let $p$ be an asymmetric seminorm on a real vector space $X$ and $\varphi: X \rightarrow \mathbb{R}$ a linear functional.

(1) The following equalities hold:

$$
\left.\left\|\left.\varphi\right|_{p}=\sup \varphi\left(B_{p}^{\prime}\right), \quad\right\| \varphi\right|_{\bar{p}}=\sup \varphi\left(B_{\bar{p}}^{\prime}\right) .
$$

Moreover, if the functional $\varphi \neq 0$ is $p$-bounded, then $\|\left.\varphi\right|_{p}>0$ and $\varphi\left(x_{0}\right)=\|\left.\varphi\right|_{p}$, for some $x_{0} \in B_{p}$, implies that $p\left(x_{0}\right)=1$.

(2) If $\varphi \neq 0$ is $(p, \bar{p})$-bounded, then

$$
\varphi\left(B_{p}^{\prime}\right)=\left(-\left.\left\|\left.\varphi\right|_{\bar{p}} ;\right\| \varphi\right|_{p}\right)
$$


If $\varphi$ is $p$-bounded but not $\bar{p}$-bounded, then

$$
\varphi\left(B_{p}^{\prime}\right)=\left(-\infty ;\left.|| \varphi\right|_{p}\right)
$$

Proof. (1) If $\left(x_{n}\right)$ is a sequence in $B_{p}$ such that $\varphi\left(x_{n}\right) \rightarrow \|\left.\varphi\right|_{p}$ for $n \rightarrow \infty$, then $x_{n}^{\prime}=(1-$ $1 / n) x_{n} \in B_{p}^{\prime}$ and $\varphi\left(x_{n}^{\prime}\right) \rightarrow \|\left.\varphi\right|_{p}$. Because

$$
\sup \varphi\left(B_{p}^{\prime}\right) \leq \sup \varphi\left(B_{p}\right)=\|\left.\varphi\right|_{p},
$$

it follows that $\sup \varphi\left(B_{p}^{\prime}\right)=\|\left.\varphi\right|_{p}$.

If $\varphi \neq 0$, and $z \in X$ is such that $\varphi(z)>0$, then the inequality $\varphi(z) \leq \|\left.\varphi\right|_{p} p(z)$ implies that $\|\left.\varphi\right|_{p}>0$.

If $x_{0} \in B_{p}$ is such that $\varphi\left(x_{0}\right)=\|\left.\varphi\right|_{p}$, then $\left.\left\|\left.\varphi\right|_{p} p\left(x_{0}\right) \geq \varphi\left(x_{0}\right)=\right\| \varphi\right|_{p}>0$, so that $p\left(x_{0}\right)>$ 0 . If $p\left(x_{0}\right)<1$, then $x_{1}=\left(1 / p\left(x_{0}\right)\right) x_{0} \in B_{p}$ and

$$
\varphi\left(x_{1}\right)=\frac{\varphi\left(x_{0}\right)}{p\left(x_{0}\right)}=\frac{\|\left.\varphi\right|_{p}}{p\left(x_{0}\right)}>\|\left.\varphi\right|_{p}=\sup \varphi\left(B_{p}\right),
$$

a contradiction.

(2) Suppose that $\varphi$ is $(p, \bar{p})$-bounded. We have

$$
\begin{aligned}
\|\left.\varphi\right|_{\bar{p}} & =\sup \{\varphi(x): \bar{p}(x)<1\}=\sup \{\varphi(-x): p(x)<1\} \\
& =-\inf \{\varphi(x): p(x)<1\} .
\end{aligned}
$$

Similar calculations show that

$$
\|\left.\varphi\right|_{\bar{p}}=-\inf \left(B_{p}\right)
$$

Because $B_{p}^{\prime}$ is convex, it follows that $\varphi\left(B_{p}^{\prime}\right)$ is an interval in $\mathbb{R}$ and

$$
\left(-\|\left.\varphi\right|_{\bar{p}} ;|\varphi|_{p}\right) \subset \varphi\left(B_{p}^{\prime}\right) \subset\left[-\left.\left\|\left.\varphi\right|_{\bar{p}} ;\right\| \varphi\right|_{p}\right] .
$$

If $\|\left.\varphi\right|_{p} \in \varphi\left(B_{p}^{\prime}\right)$, then $\|\left.\varphi\right|_{p}=\varphi\left(x_{0}\right)$, for some $x_{0} \in X$ with $p\left(x_{0}\right)<1$, in contradiction to the assertion (1) of the proposition. Similarly, if $-\|\left.\varphi\right|_{\bar{p}} \in \varphi\left(B_{p}^{\prime}\right)$, then $-\|\left.\varphi\right|_{\bar{p}}=\varphi\left(x_{1}\right)$, for some $x_{1} \in X$ with $p\left(x_{1}\right)<1$. But then, for $x_{1}^{\prime}=\left(1 / p\left(x_{1}\right)\right) x_{1} \in B_{p}$, we obtain the contradiction

$$
\varphi\left(x_{1}^{\prime}\right)=\frac{\varphi\left(x_{1}\right)}{p\left(x_{1}\right)}=\frac{-\|\left.\varphi\right|_{\bar{p}}}{p\left(x_{1}\right)}<-\|\left.\varphi\right|_{\bar{p}}=\inf \varphi\left(B_{p}\right) .
$$

If $\varphi$ is $p$-bounded but not $\bar{p}$-bounded, then

$$
\|\left.\varphi\right|_{\bar{p}}=\sup \varphi\left(B_{p}^{\prime}\right)=\infty
$$

so that, by (2.22), $\inf \varphi\left(B_{p}^{\prime}\right)=-\|\left.\varphi\right|_{\bar{p}}=-\infty$. Since $\varphi\left(B_{p}^{\prime}\right)$ is an interval in $\mathbb{R}, \|\left.\varphi\right|_{p}=$ $\sup \varphi\left(B_{p}^{\prime}\right)$, and $\|\left.\varphi\right|_{p} \notin \varphi\left(B_{p}^{\prime}\right)$, it follows that $\varphi\left(B_{p}^{\prime}\right)=\left(-\infty ; \|\left.\varphi\right|_{p}\right)$. 
Extension of bounded linear functionals. As in the symmetric case, an extension result for continuous linear functionals defined on subspaces of an asymmetric locally convex space will be particularly useful in developing a duality theory for such spaces.

Proposition 2.8. Let $(X, P)$ be an asymmetric locally convex space and $Y$ a subspace of $X$.

If $\varphi: Y \rightarrow \mathbb{R}$ is a $(P, u)$-continuous linear functional, then there exists a $(P, u)$-continuous linear functional $\Phi: X \rightarrow \mathbb{R}$ such that $\left.\Phi\right|_{Y}=\varphi$.

Proof. Suppose that the family $P$ is directed. By Proposition 2.3, there exist $p \in P$ and $L \geq 0$ such that

$$
\forall y \in Y, \quad \varphi(y) \leq L p(y)
$$

By the Hahn-Banach dominated extension theorem, there exists a linear functional $\Phi: X \rightarrow \mathbb{R}$ such that $\left.\Phi\right|_{Y}=\varphi$ and

$$
\forall x \in X, \quad \Phi(x) \leq L p(x)
$$

which, by the same Proposition 2.3, is equivalent to the $(P, u)$-continuity of $\Phi$.

The following existence result is well known in the symmetric case.

Proposition 2.9. (1) If $p$ is an asymmetric norm on a real vector space $X$ and $x_{0} \in X$ is such that $p\left(x_{0}\right)>0$, then there exists a $p$-bounded linear functional $\varphi: X \rightarrow \mathbb{R}$ such that

(i) $\varphi\left(x_{0}\right)=p\left(x_{0}\right)$,

(ii) $\left.|| \varphi\right|_{p}=1$.

(2) Let $(X, P)$ be an asymmetric locally convex space. If the topology $\tau_{P}$ is Hausdorff, then for every $x_{0} \in X, x_{0} \neq 0$, there exists $\psi \in X^{b}$ such that $\psi\left(x_{0}\right)=1$.

Proof. (1) Let $Z=\mathbb{R} x_{0}$ and $\varphi_{0}: Z \rightarrow \mathbb{R}$ be defined by $\varphi_{0}\left(t x_{0}\right)=t p\left(x_{0}\right), t \in \mathbb{R}$. Then $\varphi_{0}$ is linear and $\varphi_{0}\left(t x_{0}\right)=t p\left(x_{0}\right)=p\left(t x_{0}\right)$ for $t \geq 0$. Since $\varphi_{0}\left(t x_{0}\right)=t p\left(x_{0}\right)<0 \leq p\left(t x_{0}\right)$ for $t<0$, it follows that $\varphi_{0}(z) \leq p(z)$ for all $z \in Z$. By the Hahn-Banach extension theorem, there exists a linear functional $\varphi: X \rightarrow \mathbb{R}$ such that $\left.\varphi\right|_{Z}=\varphi_{0}$ and $\varphi(x) \leq p(x)$ for all $x \in X$, implying that $\|\left.\varphi\right|_{p} \leq 1$. Since

$$
\|\left.\varphi\right|_{p}=\sup \left\{\varphi(x): x \in B_{p}\right\} \geq \sup \left\{\varphi_{0}(z): z \in Z \cap B_{p}\right\} \geq \varphi_{0}\left(\frac{1}{p\left(x_{0}\right)} x_{0}\right)=1,
$$

it follows that $\|\left.\varphi\right|_{p}=1$.

(2) If $x_{0} \neq 0$ and $\tau_{P}$ is Hausdorff, then by Corollary 1.8, there exists $p \in P$ such that $p\left(x_{0}\right)>0$. If $\varphi: X \rightarrow \mathbb{R}$ is a $p$-bounded linear functional satisfying the conditions (i) and (ii) of the first assertion, then we can take $\psi=\left(1 / p\left(x_{0}\right)\right) \varphi$.

The $w^{b}$-topology of the dual $X^{b}$. This is the analog of the weak*-topology ( $w^{*}$-topology) on the dual of a locally convex space. In the case of an asymmetric normed space $(X, p)$, it was considered in [13].

Let $(X, P)$ be an asymmetric locally convex space and $X^{b}=X_{P}^{b}$ the asymmetric dual cone. A $w^{b}$-neighborhood of an element $\varphi \in X^{b}$ is a subset $W$ of $X^{b}$ for which there exist 
$x_{1}, \ldots, x_{n} \in X$ and $\epsilon>0$ such that

$$
V_{x_{1}, \ldots, x_{n} ; \epsilon}(\varphi):=\left\{\psi \in X^{b}: \psi\left(x_{i}\right)-\varphi\left(x_{i}\right)<\epsilon, i=1, \ldots, n\right\} \subset W
$$

For $x \in X$, define $q_{x}: X^{b} \rightarrow[0 ; \infty)$ by $q_{x}(\varphi)=\varphi(x), \varphi \in X^{b}$. Then $q_{x}$ is additive and positively homogeneous on $X^{b}$ and

$$
V_{x_{1}, \ldots, x_{n} ; \epsilon}(\varphi)=\left\{\psi \in X^{b}: q_{x_{i}}(\psi-\varphi)<\epsilon, i=1, \ldots, n\right\} .
$$

The $w^{b}$-convergence of a net $\left\{\varphi_{i}, i \in I\right\}$ to $\varphi \in X^{b}$ is equivalent to the fact that for every $x \in X$, the net $\left\{\left(\varphi_{i}-\varphi\right)(x), i \in I\right\}$ converges to 0 in $(\mathbb{R}, u)$, that is,

$$
\forall x \in X, \forall \epsilon>0, \exists i_{0} \in I \text { such that } \forall i \geq i_{0}, \quad\left(\varphi_{i}-\varphi\right)(x)<\epsilon .
$$

Since $X^{b} \subset X^{*}$ and

$$
V_{x ; \epsilon}(\varphi) \cap V_{-x ; \epsilon}(\varphi)=\left\{\psi \in X^{b}:|(\psi-\varphi)(x)|<\epsilon\right\}
$$

it follows that the $w^{b}$-topology on $X^{b}$ is induced by the $w^{*}$-topology of the space $X^{*}$.

Asymmetric polars. Let $(X, P)$ be an asymmetric locally convex space, $\left(X, P^{s}\right)$ the associated locally convex space, $X^{b}$ the asymmetric dual of $(X, P)$, and $X^{*}=\left(X, P^{s}\right)^{*}$ the conjugate space of $\left(X, P^{s}\right)$.

The polar of a nonempty subset $Y$ of $\left(X, P^{s}\right)$ is defined by

$$
Y^{\circ}=\left\{x^{*} \in X^{*}: \forall y \in Y, x^{*}(y) \leq 1\right\} .
$$

Define the corresponding set in the case of the asymmetric dual $X^{b}$ by

$$
Y^{\alpha}=Y^{\circ} \cap X^{b}=\left\{\varphi \in X^{b}: \forall y \in Y, \varphi(y) \leq 1\right\},
$$

and call it the asymmetric polar of the set $Y$.

As it is well known, the set $Y^{\circ}$ is a convex $w^{*}$-closed subset of $X^{*}$ (see, e.g., [19, page 341]). Since the $w^{b}$-topology on $X^{b} \subset X^{*}$ is induced by the $w^{*}$-topology on $X^{*}$, we have the following result.

Proposition 2.10. The asymmetric polar $Y^{\alpha}$ of a nonempty subset $Y$ of an asymmetric locally convex space $(X, P)$ is a convex $w^{b}$-closed subset of $X^{b}$.

In the following proposition, we prove the asymmetric analog of the Alaoglu-Bourbaki theorem, see, for example, [8, Theorem 4.31] or [19, Satz VIII.3.11].

Proposition 2.11. The asymmetric polar of a neighborhood of the origin of an asymmetric locally convex space $(X, P)$ is a convex $w^{b}$-compact subset of the asymmetric dual $X^{b}$.

Proof. Suppose that $P$ is directed. If $V$ is a $\tau_{P}$-neighborhood of $0 \in X$, then there exist $p \in P$ and $r>0$ such that $B_{p}(0, r) \subset V$. Because $p^{s}(x) \leq r$ implies that $p(x) \leq p^{s}(x) \leq r$, it follows that $B_{p^{s}}(0, r) \subset B_{p}(0, r) \subset V$, so that $V$ is a neighborhood of 0 in the locally convex space $(X, P)$. By the Alaoglu-Bourbaki theorem (see [19, Satz WIII.3.11]), it follows that 
$V^{\circ}$ is a convex $w^{*}$-compact subset of the dual $X^{*}$. Since $w^{b}$-compactness of $V^{\alpha}$ is equivalent to its $w^{*}$-compactness in $X^{*}$, it is sufficient to show that the set $V^{\alpha}$ is $w^{*}$-closed in $X^{*}$.

Let $\left\{\varphi_{i}: i \in I\right\}$ be a net in $V^{\alpha}$ that is $w^{*}$-convergent to $f \in X^{*}$. This means that for every $x \in X$, the net $\left\{\varphi_{i}(x): i \in I\right\}$ converges to $f(x)$ in $(\mathbb{R},|\cdot|)$. Since for every $v \in V$, $\varphi_{i}(v) \leq 1$, for all $i \in I$, it follows that $f(v) \leq 1$ for all $v \in V$. Because $f$ is linear, it is sufficient to prove its $(P, u)$-continuity at $0 \in X$. Consider for some $\epsilon>0$ the $\tau_{u}$-neighborhood $(-\infty ; \epsilon)$ of $f(0)=0 \in \mathbb{R}$. Then $U=(\epsilon / 2) V$ is a $\tau_{P}$-neighborhood of $0 \in X$, and for $v \in V$ and $u=(\epsilon / 2) v \in U$, we have

$$
f(u)=\frac{\epsilon}{2} f(v) \leq \frac{\epsilon}{2}<\epsilon,
$$

that is, $f(U) \subset(-\infty ; \epsilon)$, proving the $(P, u)$-continuity of $f$ at 0 .

It follows that $f \in V^{\alpha}$, so that $V^{\alpha}$ is $w^{*}$-closed in $X^{*}$.

\section{The continuity of the Minkowski functional and the separation of convex sets}

Proposition 2.3 can be extended to sublinear functionals.

Proposition 3.1. Let $(X, P)$ be an asymmetric locally convex space, where $P$ is a directed family of asymmetric seminorms on $X$, and let $f: X \rightarrow \mathbb{R}$ be a sublinear functional. The following assertions are equivalent.

(1) The functional $f$ is $(P, u)$-continuous at $0 \in X$.

(2) The functional $f$ is $(P, u)$-continuous on $X$.

(3) There exist $p \in P$ and $L>0$ such that

$$
\forall x \in X, \quad f(x) \leq L p(x)
$$

(4) The functional $f$ is upper semicontinuous from $\left(X, \tau_{P}\right)$ to $(\mathbb{R},|\cdot|)$.

Proof. $(1) \Rightarrow(3)$. Since $(-\infty ; 1]$ is a $\tau_{u}$-neighborhood of $f(0)=0 \in \mathbb{R}$, there exist $p \in P$ and $r>0$ such that $f\left(B_{p}(0, r)\right) \subset(-\infty ; 1]$, that is,

$$
\forall x \in X, \quad p(x) \leq r \Longrightarrow f(x) \leq 1 .
$$

By Proposition 1.4, this implies that

$$
\forall x \in X, \quad f(x) \leq \frac{1}{r} p(x) .
$$

$(3) \Rightarrow(2)$. Let $x_{0} \in X$, and for some $\epsilon>0$, let $\left(-\infty ; f\left(x_{0}\right)+\epsilon\right)$ be a $\tau_{u}$-neighborhood of $f\left(x_{0}\right)$. If $p \in P$ and $L \geq 0$ are as in the assertion (3) of the proposition, then $U=x_{0}+$ $(\epsilon /(L+1)) B_{p}$ is a $\tau_{P}$-neighborhood of $x_{0}$, and for every $z \in B_{p}$ and $x=x_{0}+(\epsilon /(L+1)) z \epsilon$ $U$, we have

$$
f(x) \leq f\left(x_{0}\right)+\frac{\epsilon}{L+1} f(z) \leq f\left(x_{0}\right)+\epsilon \frac{L}{L+1}<f\left(x_{0}\right)+\epsilon,
$$

proving the $(P, u)$-continuity of $f$ at $x_{0}$. 
Because the implication $(2) \Rightarrow(1)$ is trivial, it follows the equivalence of the first three assertions of the theorem.

As the equivalence (2) $\Leftrightarrow(4)$ holds for any mapping $f: X \rightarrow \mathbb{R}$ (see Remark 1.2), it follows the equivalence of all four assertions of the proposition.

The above proposition has the following useful corollary.

Corollary 3.2. Let $f, g$ be sublinear functionals defined on an asymmetric locally convex space $(X, P)$. If $f \leq g$ and $g$ is $(P, u)$-continuous, then $f$ is $(P, u)$-continuous too.

In particular, the result is true when $f$ is linear.

Proof. By Proposition 3.1, there exist $p \in P$ and $L \geq 0$ such that for all $x \in X, g(x) \leq$ $L p(x)$. It follows that for all $x \in X, f(x) \leq g(x) \leq L p(x)$, which, by the same proposition, implies the continuity of $f$.

Concerning the continuity of the Minkowski functional, we have the following result.

Proposition 3.3. Let $C$ be a convex absorbing subset of an asymmetric locally convex space $(X, P)$.

(1) The Minkowski functional $p_{C}$ is $(P, u)$-continuous if and only if 0 is a $\tau_{P}$-interior point of $C$.

(2) If $p_{C}$ is $(P, u)$-continuous, then

$$
\tau_{P}-\operatorname{int} C=\left\{x \in X: p_{C}(x)<1\right\} .
$$

Proof. Suppose that the family $P$ is directed.

(1) If 0 is a $\tau_{P}$-interior point of $C$, then there exist $p \in P$ and $r>0$ such that

$$
B_{p}(0, r) \subset C \subset\left\{x \in X: p_{C}(x) \leq 1\right\},
$$

that is,

$$
\forall x \in X, \quad p(x) \leq r \Longrightarrow p_{C}(x) \leq 1
$$

By Proposition 1.4, we have

$$
\forall x \in X, \quad p_{C}(x) \leq \frac{1}{r} p(x),
$$

which, by Proposition 3.1, implies the $(P, u)$-continuity of $p_{C}$.

Conversely, suppose that $p_{C}$ is $(P, u)$-continuous. Since the set $(-\infty ; 1)$ is $\tau_{u}$-open in $\mathbb{R}$, the set $\left\{x \in X: p_{C}(x)<1\right\}=p_{C}^{-1}(-\infty ; 1)$ is $\tau_{P}$-open, contains 0 , and is contained in $C$, implying that $0 \in \tau_{P}$-int $C$.

(2) If $p_{C}$ is $(P, u)$-continuous, then the above inclusion shows that

$$
\left\{x \in X: p_{C}(x)<1\right\} \subset \tau_{P}-\operatorname{int} C .
$$

If $x \in \tau_{P}$-int $C$, then there exist $p_{1} \in P$ and $r>0$ such that $B_{p_{1}}^{\prime}(x, r) \subset C$. Let $p_{2} \in P$ and $L>0$ be such that for all $x \in X, p_{C}(x) \leq L p_{2}(x)$. If $p \in P$ is such that $p \geq p_{1}, i=1,2$, then 
$B_{p}^{\prime}(x, r) \subset B_{p_{1}}^{\prime}(x, r) \subset C$ and

$$
\forall x \in X, \quad p_{C}(x) \leq L p(x) .
$$

If $p(x)=0$, then, by the above inequality, $p_{C}(x)=0<1$. If $p(x)>0$, put $x_{\alpha}=(1+\alpha) x$ for $\alpha>0$. Since $p\left(x_{\alpha}-x\right)=\alpha p(x)<r$ for $0<\alpha<r / p(x)$, it follows that $x_{\alpha} \in C$ for $0<\alpha<$ $r / p(x)$. But then, for any such $\alpha$, we have

$$
p_{C}(x)=\frac{1}{1+\alpha} p_{C}\left(x_{\alpha}\right) \leq \frac{1}{1+\alpha}<1 .
$$

The separation of convex sets. The separation results for convex subsets of locally convex spaces are key tools in the study of duality for these spaces as well as in optimization problems.

In the following two theorems, we prove the asymmetric analogs of the classical separation theorems of Eidelheit and Tukey (see [15, Theorems 2.2.26 and 2.2.28]).

Theorem 3.4. Let $(X, P)$ be an asymmetric locally convex space. If $Y_{1}, Y_{2}$ are two disjoint nonempty convex subsets of $X$ with $Y_{1} \tau_{P}$-open, then there exists a linear functional $\varphi \in X^{b}$ such that

$$
\forall y_{1} \in Y_{1}, \forall y_{2} \in Y_{2} \quad \varphi\left(y_{1}\right)<\varphi\left(y_{2}\right) .
$$

Proof. Let $y_{i}^{0} \in Y_{i}, i=1,2$, and let $x_{0}=y_{2}^{0}-y_{1}^{0}$. Since the set $Y_{1}$ is $\tau_{P}$-open and the topology $\tau_{P}$ is translation invariant, the set

$$
Y:=x_{0}+Y_{1}-Y_{2}=\cup\left\{x_{0}-y_{2}+Y_{1}: y_{2} \in Y_{2}\right\}
$$

is $\tau_{P}$-open too.

We have $0=x_{0}+y_{1}^{0}-y_{2}^{0} \in Y$ and $x_{0} \notin Y$. Indeed, if $x_{0}=x_{0}+y_{1}-y_{2}$, for some $y_{1} \in Y_{1}$ and $y_{2} \in Y_{2}$, then the element $y=y_{1}=y_{2}$ would belong to the empty set $Y_{1} \cap Y_{2}$.

By the preceding proposition, the Minkowski functional $p_{Y}$ of the $\tau_{P}$-open convex set $Y$ is sublinear, $(P, u)$-continuous, and $p_{Y}\left(x_{0}\right) \geq 1$ since $x_{0} \notin Y$. By Proposition 2.9(1), there exists a $p_{Y}$-bounded linear functional $\psi: X \rightarrow \mathbb{R}$ such that $\psi\left(x_{0}\right)=p_{Y}\left(x_{0}\right)$ and $\psi(x) \leq p_{Y}(x), x \in X$. Taking $\varphi=\left(1 / p_{Y}\left(x_{0}\right)\right) \psi$, it follows that

$$
\varphi\left(x_{0}\right)=1, \quad \forall x \in X, \quad \varphi(x)=\frac{1}{p_{Y}\left(x_{0}\right)} \psi(x) \leq \frac{1}{p_{Y}\left(x_{0}\right)} p_{Y}(x) \leq p_{Y}(x) .
$$

By Proposition 2.3, the functional $\varphi$ is $(P, u)$-continuous. Because $Y$ is $\tau_{P}$-open and $0 \in Y$, by Proposition 3.3, we have $Y=\left\{x \in X: p_{Y}(x)<1\right\}$. Since $\varphi\left(x_{0}\right)=1$, we obtain

$$
\forall y_{1} \in Y_{1}, \forall y_{2} \in Y_{2} \quad 1+\varphi\left(y_{1}\right)-\varphi\left(y_{2}\right)=\varphi\left(x_{0}+y_{1}-y_{2}\right) \leq p_{Y}\left(x_{0}+y_{1}-y_{2}\right)<1,
$$

implying that

$$
\forall y_{1} \in Y_{1}, \forall y_{2} \in Y_{2}, \quad \varphi\left(y_{1}\right)<\varphi\left(y_{2}\right)
$$


We prove now the asymmetric analog of Tukey's separation theorem.

Theorem 3.5. Let $(X, P)$ be an asymmetric locally convex space. If $Y_{1}, Y_{2}$ are two nonempty disjoint convex subsets of $X$ with $Y_{1} \tau_{P}$-compact and $Y_{2} \tau_{P}$-closed, then there exists a functional $\varphi \in X^{b}$ such that

$$
\sup \varphi\left(Y_{1}\right)<\inf \varphi\left(Y_{2}\right)
$$

Proof. Suppose that $P$ is directed.

Since $Y_{1} \cap Y_{2}=\varnothing$ and $Y_{2}$ is $\tau_{P}$-closed, for every $y \in Y_{1}$, there exist $p_{y} \in P$ and $r_{y}>0$ such that

$$
\left(y+2 r_{y} B_{p_{y}}^{\prime}\right) \cap Y_{2}=\varnothing .
$$

The $\tau_{P}$-open cover $\left\{y+r_{y} B_{p_{y}}^{\prime}: y \in Y_{1}\right\}$ of the $\tau_{P}$-compact set $Y_{1}$ contains a finite subcover $\left\{y_{k}+r_{k} B_{p_{k}}^{\prime}: k=1,2, \ldots, n\right\}$, where $p_{k}=p_{y_{k}}$ and $r_{k}=r_{y_{k}}$ for $k=1, \ldots, n$. Take $p \in P$ such that $p \geq p_{k}, k=1,2, \ldots, n$, put $r:=\min \left\{r_{k}: k=1,2, \ldots, n\right\}$ and show that

$$
\left(Y_{1}+r B_{p}^{\prime}\right) \cap Y_{2}=\varnothing .
$$

Indeed, if $y^{\prime}=y+r u$ for some $y \in Y_{1}, u \in B_{p}^{\prime}$, and $y^{\prime} \in Y_{2}$, then, choosing $k \in\{1,2, \ldots, n\}$ such that $y \in y_{k}+r_{k} B_{p_{k}}^{\prime}$, we have

$$
y^{\prime}=y+r u \in y_{k}+r B_{p}^{\prime}+r_{k} B_{p_{k}}^{\prime} \subset y_{k}+r_{k} B_{p_{k}}^{\prime}+r_{k} B_{p_{k}}^{\prime}=y_{k}+2 r_{k} B_{p_{k}}^{\prime},
$$

in contradiction to $(3.18)$.

The set $Z:=Y_{1}+r B_{p}^{\prime}$ is convex, $\tau_{P}$-open, and disjoint from $Y_{2}$. By Theorem 3.4, there exists $\varphi \in X^{b}$ such that

$$
\forall y \in Y_{1}, \forall u \in B_{p}^{\prime}, \forall y^{\prime} \in Y_{2} \quad \varphi(y)+r \varphi(u)<\varphi\left(y^{\prime}\right) .
$$

By Proposition 2.3, there exist $q_{1} \in P$ and $L>0$ such that for all $x \in X, \varphi(x) \leq L q_{1}(x)$. If $q \in P$ is such that $q \geq \max \left\{p, q_{1}\right\}$, then $\varphi(x) \leq L q(x), x \in X$, and $B_{q}^{\prime} \subset B_{p}^{\prime}$, so that

$$
\forall y \in Y_{1}, \forall u \in B_{q}^{\prime}, \forall y^{\prime} \in Y_{2} \quad \varphi(y)+r \varphi(u)<\varphi\left(y^{\prime}\right) .
$$

By (3.21), $\varphi \neq 0$, so that by Proposition 2.7, $\|\left.\varphi\right|_{q}=\sup \varphi\left(B_{q}^{\prime}\right)>0$. Passing in (3.22) to supremum with respect to $u \in B_{q}^{\prime}$, we get

$$
\forall y \in Y_{1}, \forall y^{\prime} \in Y_{2} \quad \varphi(y)+r \|\left.\varphi\right|_{q} \leq \varphi\left(y^{\prime}\right),
$$

implying that

$$
r \|\left.\varphi\right|_{q}+\sup \varphi\left(Y_{1}\right) \leq \inf \varphi\left(Y_{2}\right)
$$

It follows that

$$
\sup \varphi\left(Y_{1}\right)<\inf \varphi\left(Y_{2}\right)
$$


Remark 3.6. The inequality in Theorem 3.4 cannot be reversed, in the sense that, under the same hypotheses on the sets $Y_{1}$ and $Y_{2}$, we cannot find a $(P, u)$-continuous linear functional $\psi$ on $X$ such that

$$
\forall y_{2} \in Y_{2}, \forall y_{1} \in Y_{1} \quad \psi\left(y_{2}\right)<\psi\left(y_{1}\right)
$$

This is due, on one side, to the fact that the functional $-\varphi$ need not be $(P, u)$-continuous, where $\varphi$ is the linear functional given by Theorem 3.4. On the other side, analyzing the proof of Theorem 3.4, it follows that we should work with the set $Y^{\prime}:=x_{0}+Y_{2}-Y_{1}$ which need not be $\tau_{P}$-open, because the $\tau_{P}$-openness of $Y_{1}$ does not imply the $\tau_{P}$-openness of $-Y_{1}$, see Example 1.3.

The same caution must be taken when applying Theorem 3.5.

Extreme points and the Krein-Milman theorem. We start by recalling some notions and facts. A point $e$ of a convex subset of a vector space $X$ is called an extreme point of $Y$ provided that $(1-t) x+t y=e$, for some $x, y \in Y$ and $0<t<1$, implies that $x=y=e$. A nonempty convex subset $Z$ of $Y$ is called an extremal subset of $Y$ if $(1-t) x+t y \in Z$, for some $x, y \in Y$ and $0<t<1$, implies that $x, y \in Z$ (in fact, $[x ; y] \subset Z$, by the convexity of $Z$ ). Obviously, that a one-point set $Z=\{e\}$ is an extremal subset of $Y$ if and only if $e$ is an extreme point of $Y$. Also, if $W$ is an extremal subset of the extremal subset $Z$ of $Y$, then $W$ is an extremal subset of $Y$ too. In particular, if $e$ is an extreme point of an extremal subset $Z$ of $Y$, then $e$ is an extreme point of $Y$. The intersection of a family of extremal subsets of $Y$ is an extremal subset of $Y$ provided that it is nonempty. We denote by ext $Y$ the (possibly empty) set of extreme points of the convex set $Y$.

The following proposition is an immediate consequence of the definitions.

Proposition 3.7. Let $Y$ be a nonempty convex subset of a vector space $X$ and $f$ a linear functional on $X$. If the set $Z=\{z \in Y: f(z)=\sup f(Y)\}$ is nonempty, then it is an extremal subset of $Y$. A similar assertion holds for the set $W=\{w \in Y: f(w)=\inf f(Y)\}$.

We can state and prove now the Krein-Milman theorem in the asymmetric case.

Theorem 3.8. Let $(X, P)$ be an asymmetric locally convex space such that the topology $\tau_{P}$ is Hausdorff. Then any nonempty convex $\tau_{P}$-compact subset $Y$ of $X$ coincides with the $\tau_{P-}$ closed convex hull of the set of its extreme points

$$
Y=\tau_{P}-\mathrm{cl}-\operatorname{co}(\operatorname{ext} Y)
$$

Proof. All the topological notions will concern the $\tau_{P}$-topology of $X$ so that we will omit " $\tau_{P^{-}}$" in the following. By Proposition 2.9(2), for every $x \in X, x \neq 0$, there exists $\varphi \in X^{b}$ with $\varphi(x)=1$.

Fact 3.9. Every nonempty convex compact subset $Z$ of $X$ has an extreme point.

Let

$$
\mathscr{F}:=\{F: F \text { is a closed extremal subset of } Z\} \text {, }
$$


and define the order in $\mathscr{F}$ by $F_{1} \leq F_{2} \Leftrightarrow F_{1} \subset F_{2}$ and show that the set $\mathscr{F}$ is nonempty and downward inductively ordered. Because $Y$ is $\tau_{P}$-compact and the topology $\tau_{P}$ is Hausdorff, it follows that $Y$ is convex and $\tau_{P}$-closed, so that $Y \in \mathscr{F}$. Since a totally ordered subfamily $\mathscr{G}$ of $\mathscr{F}$ has the finite intersection property, by the compactness of the set $Z$, the set $G=\cap \mathscr{G}$ is nonempty, closed, and extremal. Therefore, $G \in \mathscr{F}$ is a lower bound for $\mathscr{G}$. By Zorn's lemma, the ordered set $\mathscr{F}$ has a minimal element $F_{0}$. If we show that $F_{0}$ is a one-point set, $F_{0}=\left\{x_{0}\right\}$, then $x_{0}$ will be an extreme point of $Z$.

Suppose that $F_{0}$ contains two distinct points $x_{1}, x_{2}$, and let $p \in P$ be such that $p\left(x_{1}-\right.$ $\left.x_{2}\right)>0$. Let $\varphi$ be a $p$-bounded linear functional such that $\varphi\left(x_{1}-x_{2}\right)=p\left(x_{1}-x_{2}\right)>0$ (see Proposition 2.9(1)). It follows that $\varphi \in X^{b}$, so that $\varphi$ is upper semicontinuous as a mapping from $\left(X, \tau_{P}\right)$ to $(\mathbb{R},|\cdot|)$. By the compactness of the set $F_{0}$, the set

$$
F_{1}=\left\{x \in F_{0}: \varphi(x)=\sup \varphi\left(F_{0}\right)\right\}=\left\{x \in F_{0}: \varphi(x) \geq \sup \varphi\left(F_{0}\right)\right\}
$$

is nonempty and closed. By Proposition 3.7, $F_{1}$ is an extremal subset of $F_{0}$, thus an extremal subset of $Z$. Therefore, $F_{1} \in \mathscr{F}, F_{1} \subset F_{0}$, and $x_{2} \in F_{0} \backslash F_{1}$ in contradiction to the minimality of $F_{0}$.

Fact 3.10. $Y=\tau_{P}-\mathrm{clco}(\operatorname{ext} Y)$.

The inclusion $\operatorname{ext}(Y) \subset Y$ implies that $Y_{1}:=\tau_{P}-\operatorname{clco}(\operatorname{ext} Y) \subset Y$. As a closed subset of a compact set, the set $Y_{1}$ is convex and compact. Supposing that there exists a point $y_{0} \in Y \backslash Y_{1}$, then, by Theorem 3.5, there exists $\varphi \in X^{b}$ such that

$$
\sup \varphi\left(Y_{1}\right)<\varphi\left(y_{0}\right)
$$

Using again the upper semicontinuity of $\varphi$ as a mapping from $\left(X, \tau_{P}\right)$ to $(\mathbb{R},|\cdot|)$, we see that the set

$$
F=\{y \in Y: \varphi(y)=\sup \varphi(Y)\}=\{y \in Y: \varphi(y) \geq \sup \varphi(Y)\}
$$

is nonempty, convex, and compact, so that, by Fact 3.9, it has an extreme point $e_{1}$. Since $F$ is an extremal subset of $Y$, it follows that $e_{1}$ is an extreme point of $Y$, implying that $e_{1} \in Y_{1}$. Taking into account (3.30), we obtain the contradiction

$$
\sup \varphi(Y)=\varphi\left(e_{1}\right) \leq \sup \varphi\left(Y_{1}\right)<\varphi\left(y_{0}\right) \leq \sup \varphi(Y)
$$

\section{The asymmetric weak topology}

The weak topology of a locally convex space $(X, Q)$ is defined by the locally convex basis $\mathcal{W}$ formed by the sets of the form

$$
V_{x_{1}^{*}, \ldots, x_{n}^{*} ; \epsilon}^{\prime}=\left\{x \in X:\left|x_{i}^{*}(x)\right|<\epsilon, 1 \leq i \leq n\right\},
$$

for $n \in \mathbb{N}, x_{1}^{*}, \ldots, x_{n}^{*} \in X^{*}$ and $\epsilon>0$. Obviously, we can suppose that $x_{i}^{*} \neq 0, i=1, \ldots, n$.

The duality theory for locally convex spaces is based on the following key lemma of algebraic nature. 
Lemma 4.1 (see [19, Lemma VIII.3.3]). Let $X$ be a vector space and $f, f_{1}, \ldots, f_{n}: X \rightarrow \mathbb{R}$ linear functionals. The following assertions are equivalent.

(1) $f \in \operatorname{sp}\left\{f_{1}, \ldots, f_{n}\right\}$.

(2) There exists $L \geq 0$ such that

$$
\forall x \in X, \quad f(x) \leq L \max \left\{\left|f_{1}(x)\right|, \ldots,\left|f_{n}(x)\right|\right\} .
$$

(3) $\bigcap_{i=1}^{n} \operatorname{ker} f_{i} \subset \operatorname{ker} f$.

In our case, this lemma takes the form.

Lemma 4.2. Let $f, f_{1}, \ldots, f_{n}$ be real linear functionals on a vector space $X$, with $f_{1}, \ldots, f_{n}$ linearly independent. Then the following assertions are equivalent.

(1) For all $x \in X, f_{i}(x) \leq 0, i=1, \ldots, n \Rightarrow f(x) \leq 0$.

(2) There exists $L \geq 0$ such that for all $x \in X, f(x) \leq L \max \left\{f_{i}(x): 1 \leq i \leq n\right\}$.

(3) There exist $a_{1}, \ldots, a_{n} \geq 0$, such that $f=\sum_{i=1}^{n} a_{i} f_{i}$.

Proof. Since the implications $(2) \Rightarrow(1)$ and $(3) \Rightarrow(2)$ are obvious, it is sufficient to prove $(1) \Rightarrow(3)$.

If $f_{i}(x)=0$ for $i=1, \ldots, n$, then $f_{i}(-x)=-f_{i}(x)=0, i=1, \ldots, n$, so that $f(x) \leq 0$ and $-f(x)=f(-x) \leq 0$, implying that $f(x)=0$. Therefore Lemma 4.1(3) is fulfilled, so that there exist $a_{1}, \ldots, a_{n} \in \mathbb{R}$ such that $f=\sum_{i=1}^{n} a_{i} f_{i}$. It remains to show that $a_{j} \geq 0$ for $j=$ $1, \ldots, n$. Because $f_{1}, \ldots, f_{n}$ are linearly independent, there exist the elements $x_{j} \in X$ such that $f_{i}\left(x_{j}\right)=-\delta_{i j} \leq 0, i, j=1,2, \ldots, n$, where $\delta_{i j}$ is the Kronecker symbol. It follows that $f\left(x_{j}\right) \leq 0$ and

$$
-a_{j}=\sum_{i=1}^{n} a_{i} f_{i}\left(x_{j}\right)=f\left(x_{j}\right) \leq 0,
$$

for $j=1, \ldots, n$

Define the asymmetric weak topology $w^{\alpha}$ on an asymmetric locally convex space $(X, P)$ as the asymmetric locally convex topology generated by the asymmetric locally convex basis $\mathcal{W}_{\alpha}^{\prime}$ formed by the sets

$$
V_{\varphi_{1}, \ldots, \varphi_{n} ; \epsilon}^{\prime}=\left\{x \in X: \varphi_{i}(x)<\epsilon, 1 \leq i \leq n\right\}
$$

for $n \in \mathbb{N}, \varphi_{1}, \ldots, \varphi_{n} \in X^{b}$ and $\epsilon>0$. The neighborhoods of an arbitrary point $x \in X$ are subsets of $X$ containing a set of the form $x+V_{\varphi_{1}, \ldots, \varphi_{n} ; \epsilon}^{\prime}=\left\{x^{\prime} \in X: \varphi_{i}\left(x^{\prime}-x\right)<\epsilon, 1 \leq i \leq\right.$ $n\}$.

The sets

$$
V_{\varphi_{1}, \ldots, \varphi_{n} ; \epsilon}=\left\{x \in X: \varphi_{i}(x) \leq \epsilon, 1 \leq i \leq n\right\}
$$

generate the same topology.

In the following proposition, we collect some properties of the topology $w^{\alpha}$. 
Proposition 4.3. Let $(X, P)$ be an asymmetric locally convex space and $X^{b}=(X, P)^{b}$ its asymmetric dual cone.

(1) The topology $\tau_{P}$ is finer than $w^{\alpha}$.

(2) For $\varphi \in X^{b}$ and $\epsilon>0$, the set $\{x \in X: \varphi(x)<\epsilon\}$ is $w^{\alpha}$-open and $\{x \in X: \varphi(x) \geq \epsilon\}$ is $w^{\alpha}$-closed.

(3) A net $\left\{x_{i}: i \in I\right\}$ in $X$ is $w^{\alpha}$-convergent to $x \in X$ if and only if for every $\varphi \in X^{b}$, the net $\left\{\varphi\left(x_{i}\right)\right\}$ converges to $\varphi(x)$ in $(\mathbb{R}, u)$. This means the following:

$$
\forall \varphi \in X^{b}, \forall \epsilon>0, \exists i_{0} \text { such that } \forall i \geq i_{0}, \quad \varphi\left(x_{i}-x\right)<\epsilon .
$$

(4) The asymmetric dual $\left(X, w^{\alpha}\right)^{b}$ of the asymmetric locally convex space $\left(X, w^{\alpha}\right)$ agrees with $X^{b}$.

Proof. Suppose that $P$ is directed.

(1) Let $V^{\prime}=V_{\varphi_{1}, \ldots, \varphi_{n} ; \epsilon}^{\prime}$ be an element of the locally convex basis (4.4). Because $\varphi_{i}$ are $(P, u)$-continuous, there exist $p_{i} \in P$ and $L_{i} \geq 0$ such that

$$
\forall x \in X, \quad \varphi_{i}(x) \leq L_{i} p_{i}(x), \quad \text { for } i=1, \ldots, n .
$$

The multiball $U^{\prime}=\left\{x \in X: p_{i}(x)<\epsilon /(L+1), 1 \leq i \leq n\right\}$, where $L=\max L_{i}$, is contained in $V^{\prime}$, showing that $V^{\prime}$ is a $\tau_{P}$-neighborhood of $0 \in X$.

(2) If $V=\{x \in X: \varphi(x)<\epsilon\}$ and $x_{0} \in V$, then the $w^{\alpha}$-neighborhood $\{x \in X: \varphi(x-$ $\left.\left.x_{0}\right)<\epsilon-\varphi\left(x_{0}\right)\right\}$ of $x_{0}$ is contained in $V^{\prime}$ because

$$
\varphi\left(x-x_{0}\right)<\epsilon-\varphi\left(x_{0}\right) \Longrightarrow \varphi(x)=\varphi\left(x-x_{0}\right)+\varphi\left(x_{0}\right)<\epsilon \text {. }
$$

The assertion (3) follows from definitions.

(4) Because $\tau_{P}$ is finer than $w^{\alpha}$, the identity map Id $:\left(X, \tau_{P}\right) \rightarrow\left(X, w^{\alpha}\right)$ is continuous, implying the $(P, u)$-continuity of $\varphi \circ$ Id for any $\varphi \in\left(X, w^{\alpha}\right)^{b}$, that is, $\left(X, w^{\alpha}\right)^{b} \subset(X, P)^{b}$.

Conversely, if $\varphi$ is a $(P, u)$-continuous linear functional, then the set $V=\{x \in X$ : $\varphi(x)<\epsilon\}$ is a $w^{\alpha}$-neighborhood of $0 \in X$ and $\varphi(V) \subset(-\infty ; \epsilon)$ for every $\epsilon>0$, proving the $\left(w^{\alpha}, \tau_{u}\right)$-continuity of $\varphi$ at 0 , and by the linearity of $\varphi$, on the whole $X$.

As in the symmetric case, the closed convex sets are the same for the topologies $\tau_{P}$ and $w^{\alpha}$.

Proposition 4.4. Let $(X, P)$ be an asymmetric locally convex space and $Y$ a convex subset of $X$. Then $Y$ is $w^{\alpha}$-closed if and only if it is $\tau_{P}$-closed.

Proof. Because $\tau_{P}$ is finer than $w^{\alpha}$, it follows that any (not necessarily convex) $w^{\alpha}$-closed subset of $X$ is also $\tau_{P}$-closed.

Suppose now that the convex set $Y$ is $\tau_{P}$ but not $w^{\alpha}$-closed. If $x_{0}$ is a point in $w^{\alpha}-\mathrm{cl} Y \backslash$ $Y$, then, applying Theorem 3.5 to the sets $\left\{x_{0}\right\}$ and $Y$, we get a functional $\varphi \in X^{b}$ such that

$$
\varphi\left(x_{0}\right)<\inf \varphi(Y)
$$


If $m:=\inf \varphi(Y)$, then $V=\left\{x \in X: \varphi\left(x-x_{0}\right)<2^{-1}\left(m-\varphi\left(x_{0}\right)\right)\right\}$ is a $w^{\alpha}$-neighborhood of $x_{0}$. Because

$$
\varphi(x)=\varphi\left(x-x_{0}\right)+\varphi\left(x_{0}\right)<\frac{m+\varphi\left(x_{0}\right)}{2}<m,
$$

for every $x \in V$, it follows that $V \cap Y=\varnothing$, in contradiction to $x_{0} \in w^{\alpha}$-cl $Y$.

The proposition has the following corollary.

Corollary 4.5. Let $(X, P)$ be an asymmetric locally convex space. Then for every subset $Z$ of $X$, the following equality holds:

$$
w^{\alpha}-\operatorname{clco}(Y)=\tau_{P}-\operatorname{clco}(Y) \text {. }
$$

Proof. By the definition of the closed convex hull and the preceding proposition, we have the equalities

$$
\begin{aligned}
w^{\alpha}-\operatorname{clco}(Y) & =\bigcap\left\{Y: Y \subset X, Y \text { convex and } w^{\alpha} \text {-closed }\right\} \\
& =\bigcap\left\{Y: Y \subset X, Y \text { convex and } \tau_{P} \text {-closed }\right\} \\
& =\tau_{P}-\operatorname{clco}(Y) .
\end{aligned}
$$

Remark 4.6. We can define the asymmetric polar of a subset $W$ of the dual $X^{b}$ of an asymmetric locally convex space $(X, P)$ by

$$
W_{\alpha}=\{x \in X: \forall \varphi \in W, \varphi(x) \leq 1\} .
$$

Since, for $\varphi \in X^{b}$, a set of the form $\{x \in X: \varphi(x) \leq 1\}$ is not necessarily $\tau_{P}$-closed, the set $W_{\alpha}$ need not be $\tau_{P}$-closed. Therefore, an asymmetric analog of the bipolar theorem (see [19, Satz WIII.3.9]), asserting that

$$
\left(A^{\circ}\right)_{\circ}=-\operatorname{clco}(A \cup\{0\})
$$

for any subset $A$ of a locally convex space $(X, Q)$, does not hold in the asymmetric case.

\section{References}

[1] C. Alegre, J. Ferrer, and V. Gregori, Quasi-uniformities on real vector spaces, Indian J. Pure Appl. Math. 28 (1997), no. 7, 929-937.

[2] On the Hahn-Banach theorem in certain linear quasi-uniform structures, Acta Math. Hungar. 82 (1999), no. 4, 325-330.

[3] A. R. Alimov, The Banach-Mazur theorem for spaces with nonsymmetric distance, Uspekhi Mat. Nauk 58 (2003), no. 2(350), 159-160 (Russian), translated in Russian Math. Surveys 58 (2003), no. 2, 367-369.

[4] A. Andrikopoulos, Completeness in quasi-uniform spaces, Acta Math. Hungar. 105 (2004), no. 12, 151-173.

[5] P. A. Borodin, The Banach-Mazur theorem for spaces with an asymmetric norm and its applications in convex analysis, Mat. Zametki 69 (2001), no. 3, 329-337 (Russian), translated in Math. Notes 69 (2001), no. 3-4, 298-305. 


\section{Asymmetric locally convex spaces}

[6] S. Cobzaş, Separation of convex sets and best approximation in spaces with asymmetric norm, Quaest. Math. 27 (2004), no. 3, 275-296.

[7] S. Cobzaş and C. Mustăţa, Extension of bounded linear functionals and best approximation in spaces with asymmetric norm, Rev. Anal. Numér. Théor. Approx. 33 (2004), no. 1, 39-50.

[8] M. Fabian, P. Habala, P. Hájek, V. Montesinos Santalucía, J. Pelant, and V. Zizler, Functional Analysis and Infinite-Dimensional Geometry, CMS Books in Mathematics/Ouvrages de Mathématiques de la SMC, vol. 8, Springer, New York, 2001.

[9] J. Ferrer, V. Gregori, and C. Alegre, Quasi-uniform structures in linear lattices, Rocky Mountain J. Math. 23 (1993), no. 3, 877-884.

[10] P. Fletcher and W. F. Lindgren, Quasi-Uniform Spaces, Lecture Notes in Pure and Applied Mathematics, vol. 77, Marcel Dekker, New York, 1982.

[11] L. M. García-Raffi, S. Romaguera, and E. A. Sánchez Pérez, Sequence spaces and asymmetric norms in the theory of computational complexity, Math. Comput. Modelling 36 (2002), no. 1$2,1-11$.

[12] _ On Hausdorff asymmetric normed linear spaces, Houston J. Math. 29 (2003), no. 3, $717-728$.

[13] - The dual space of an asymmetric normed linear space, Quaest. Math. 26 (2003), no. 1, 83-96.

[14] Weak topologies on asymmetric normed linear spaces and non-asymptotic criteria in the theory of complexity analysis of algorithms, J. Anal. Appl. 2 (2004), no. 3, 125-138.

[15] R. E. Megginson, An Introduction to Banach Space Theory, Graduate Texts in Mathematics, vol. 183, Springer, New York, 1998.

[16] C. Mustăţa, A Phelps type theorem for spaces with asymmetric norms, Bul. Ştiinţ. Univ. Baia Mare Ser. B Fasc. Mat.-Inform. 18 (2002), no. 2, 275-280.

[17] On the uniqueness of the extension and unique best approximation in the dual of an asymmetric normed linear space, Rev. Anal. Numér. Théor. Approx. 32 (2003), no. 2, 187192.

[18] S. Romaguera and M. Schellekens, Duality and quasi-normability for complexity spaces, Appl. Gen. Topol. 3 (2002), no. 1, 91-112.

[19] D. Werner, Funktionalanalysis [Functional Analysis], 3rd, revised and extended ed., Springer, Berlin, 2000.

S. Cobzaş: Department of Mathematics, Faculty of Mathematics and Computer Science, BabeşBolyai University, 400084 Cluj-Napoca, Romania

E-mail address: scobzas@math.ubbcluj.ro 


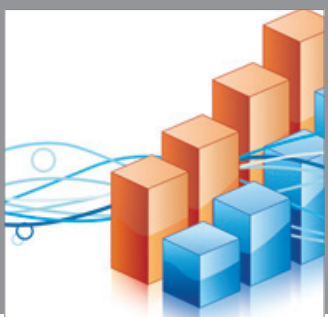

Advances in

Operations Research

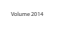

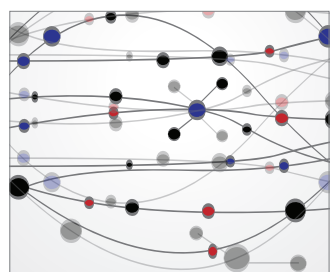

\section{The Scientific} World Journal
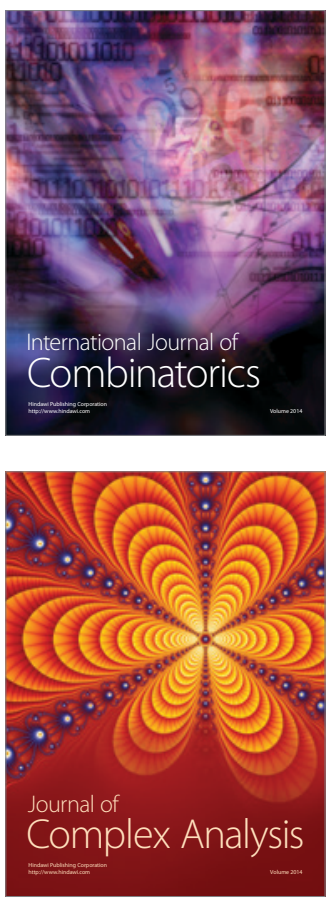

International Journal of

Mathematics and

Mathematical

Sciences
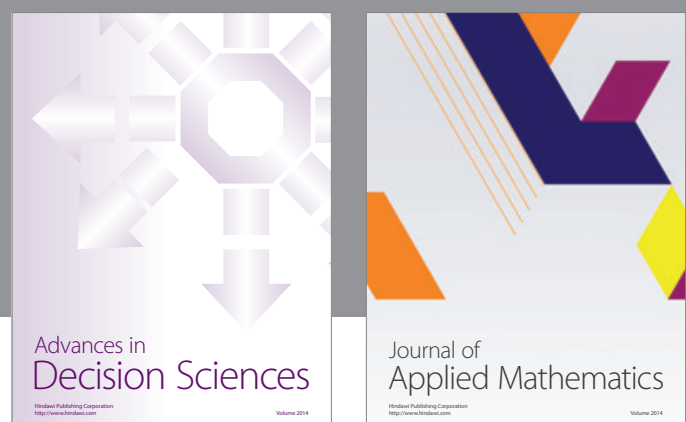

Journal of

Applied Mathematics
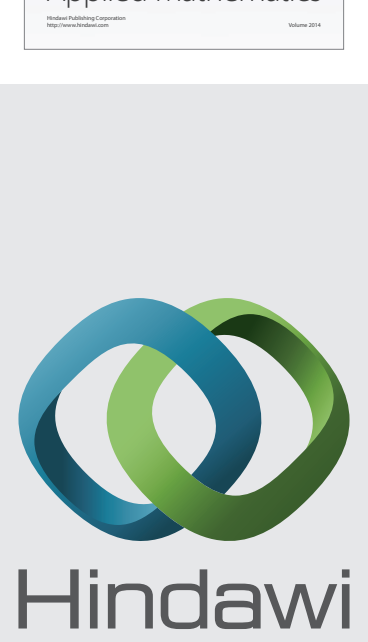

Submit your manuscripts at http://www.hindawi.com
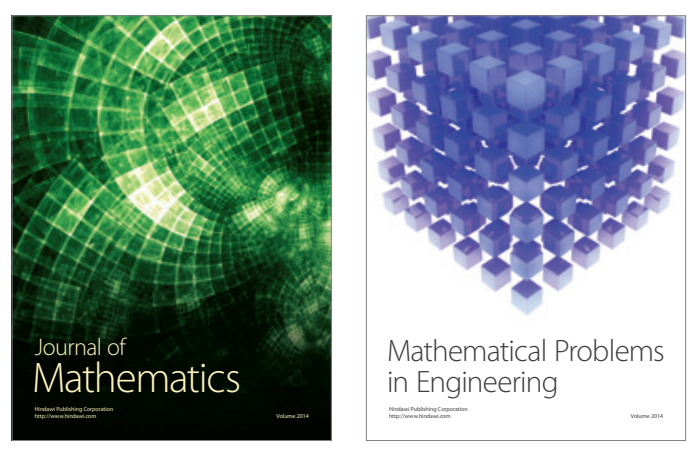

Mathematical Problems in Engineering
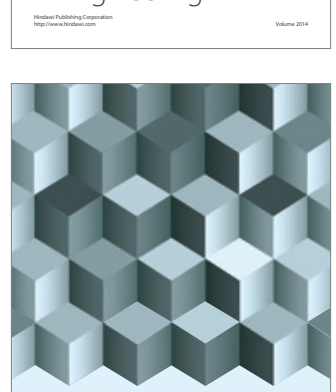

Journal of

Function Spaces
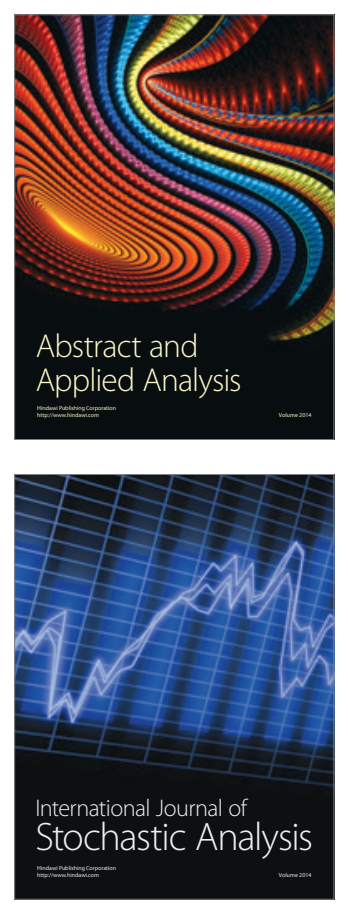

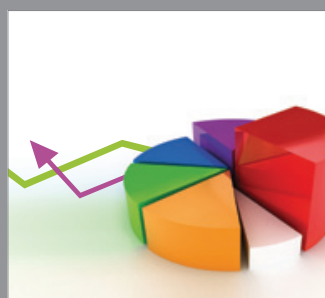

ournal of

Probability and Statistics

Promensencen
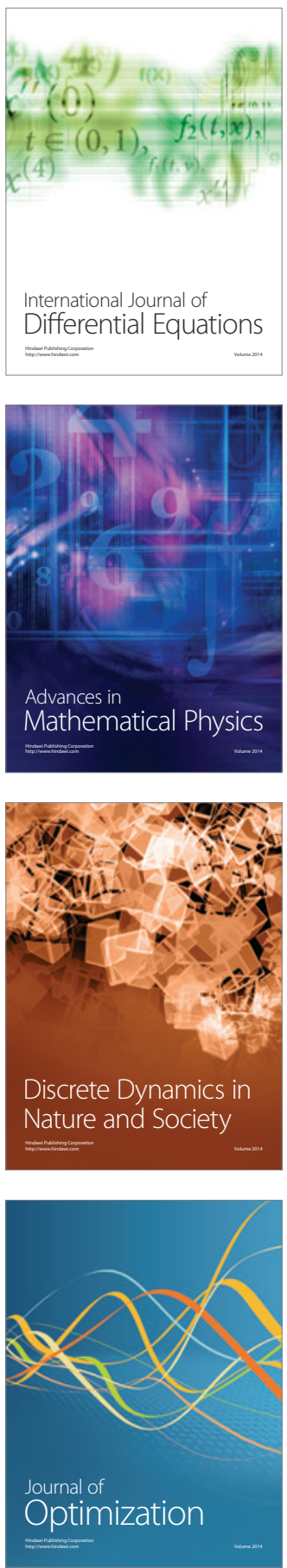